\title{
Some Characteristics of Human Problem-Solving in Chess ${ }^{1}$
}

\author{
DANIEL A. WAGNeR \\ The University of Michigan
}

AND

Martin J. Scurrah

E.S.A.N.

\begin{abstract}
Following the design of de Groot (1965) and Newell and Simon (1965), two Ss were asked to produce a total of six verbal chess protocols over a variety of conditions involving: middle and end game positions; real and artificial board positions; and differing time constraints. The analysis of these protocols yielded problem-solving episodes similar to those described by Newell and Simon. To account for these data, a modification of the Newell and Simon model was developed. The modified model gives a good account both of the data obtained in this study and also those described by Newell and Simon.
\end{abstract}

The rebirth of the chess problem as a tool for studying complex human thinking has two main causes: First, the chess game is precisely the type of problem that pushes human cognitive capacity to its limit, forcing the human to choose among a large number of alternatives with limited storage capacity; and second, the chess game is well-defined in that the objects (the chess pieces) and the primitive operators (the moves) are known, and thus may be identified in verbal protocols with a minimum loss of information. The first major modern study of problemsolving in chcss was by do Groot (1065). He presented his subjects (Ss) with a series of board positions taken from tournament and private games, and asked each $S$ to analyze a preset board situation by verbaliz-

\footnotetext{
${ }^{1}$ Requests for reprints should be sent to Daniel A. Wagner, The University of Michigan, Department of Psychology, Human Performance Center, 330 Packard Street, Ann Arbor, MI. 48104. M. J. Scurrah is a Tufts University Latin American Teaching Fellow at the Escuela de Administracion de Negocios Para Graduados, Lima, Peru. The authors would like to express their appreciation to William H. Starbuck, Allen Newell, Robert K. Lindsay, and James G. Greeno for comments and suggestions on earlier drafts of this paper. The authors wish to thank Steve Fellner and Paul Joss who freely devoted their time as subjects for this study. The research and original draft were completed at Cornell University.
} 
ing his thoughts. The resulting protocol was recorded by hand. De Groot found a general chess problem-solving process, which he called "progressive deepening," whereby promising move sequences were investigated in greater depth until a choice could be made. Newell and Simon (1965) followed the method of de Groot, with the aid of a tape recorder, in order to further specify and detail "progressive deepening." Using one $S$ with one of de Groot's chess positions, they found that the obtained protocol could be divided into "episodes" or individual problem-solving scenarios. Further, they were able to posit a set of rules to describe a substantial portion of their S's protocol.

The present study uses the same procedures as Newell and Simon (1965), but applies them to a broader range of situations. Specifically, we study: the stability of one $S$ over five different board positions; differences, if any, between artificial board situations and real game situations; differences under varying time constraints; and the stability of human chess play characteristics over three $S_{s}$ (including the $S$ of Newell and Simon).

\section{METHOD}

In general, the method used follows that of Newell and Simon. The S entered a room, was presented an artificial (i.e., previously constructed) board position, and was instructed as follows:

In a moment I shall show you a position taken from an actual tournament game. You get the side of the player on move [who moves next]. You are requested to think of a move and then to play it on the board as if the position had arisen during one of your own tournament games. In addition, however, I should like you to do all of your thinking out loud, insofar as is possible, so that I can follow and record the way in which you arrive at your move. The idea is for me to be able to study the thought process as it develops, so no special achievement is expected of you. Please voice everything that comes into your head regardless of its correctness. I should like to follow the course of your thought, so the unsuccessful tries and variations that you may later find are just as important to me as the correct ones [de Groot, 1965, p. 96].

The entire protocol was tape recorded.

Four protocols were obtained from $S 1$, a graduate student in mathematics, and a class $\mathrm{C}$ chess player. There were variations in the types of board positions and in time constraints. Positions A (de Groot, 1965, p. 89) and F (Fine, 1952, p. 439) were middle game positions. Position A was that used by Newell and Simon, and had no time constraint. Position F had a 7 minute time limit. Positions B (de Groot, 1965, p. 91) and E (Fine, 1952, Position PB-6) were end game positions, and had time limits of 10 and 20 minutes, respectively. 
The final two protocols, $\mathrm{C}$ and $\mathrm{D}$, were obtained to test whether differences appear between the above artificial or constructed board positions and a real game situation. S1 faced an actual opponent and developed his position himself, rather than having it presented to him. S1 was therefore matched with another $S, S 2$, a graduate student in astronomy, and also a class $\mathrm{C}$ chess player. After the 12th move, play was stopped and $S 2$ left the room while $S 1$ analyzed his position (Protocol C). When S1 had made his move, he left the room and S2 returned and analyzed the new board situation (Protocol D). Both Ss had 15 minute time limits.

\section{Definitions}

Terms used in analyzing the protocols are defined as follows.

Move:

The proposed movement by either player of one of his pieces from one square to another, either by occupying an empty square or by capturing a piece (e.g., $\mathrm{N} \times \mathrm{B}^{\prime}$ ).

Base move: An initial move proposed in the context of a given board position; the first move in any episode.

Episode:

$\mathrm{A}$ base move and the sequence of moves generated in response to that base move.

Depth: $\quad$ The minimum number of moves required to reach a particular point from the base move.

Line: $\quad$ The sequence of moves leading to the last evaluation made in an episode.

Branch: Any move or sequence of moves, not included in the line, that leads to an evaluation.

Evaluation: The S's estimate, in terms of his objectives, of the chess situation at the end of a line or branch.

New move: A move that has not been proposed in the previous branch or line at the same depth.

Different move: A move that has not been previously proposed in any previous branch or line at the same depth.

Typical Chess Abbreviations:

$\mathrm{K}=$ King, $\mathrm{Q}=$ Queen, $\mathrm{R}=$ Rook, $\mathrm{B}=$ Bishop, $\mathrm{N}=$ Knight, $\mathrm{P}=$ Pawn.

$\mathrm{Q} \times \mathrm{R}^{\prime}$ means white Queen captures black (') Rook.

Q-Q4 means white Queen moves to the position three spaces in front of the initial $Q$ position. 


\section{RESULTS AND DISCUSSION}

\section{The Problem Behavior Graph}

After transcribing the tapes, the protocols were analyzed in the manner of Newell and Simon (1965). All protocols fell naturally into episodes, although we found it convenient to define an episode slightly differently from Newell and Simon's definition. We defined an episode as a base move and the sequence of moves generated in response to that base move. The only difference between our definition and Newell and Simon's is that they allowed a new episode to begin without returning to a base move where sufficient discontinuity occurred; we always required that there be a return to a base move. Thus, Newell and Simon included as separate episodes five that we would have included in other episodes. A representation of the entire set of such episodes is called a Problem Behavior Graph (PBG; Newell, 1968). The board for Position

TABLE 1

Summary of Protocol Statistics

\begin{tabular}{|c|c|c|c|c|c|c|c|}
\hline & \multicolumn{7}{|c|}{ Protocols } \\
\hline & $\mathrm{A}(S 1)$ & $\mathrm{A}(S 0)$ & $\mathrm{B}(S 1)$ & $\mathrm{C}(S 1)$ & $\mathrm{D}(S 2)$ & $\mathrm{E}(\mathrm{S} 1)$ & $\mathrm{F}(S 1)$ \\
\hline Type of game & middle & middle & end & middle & middle & end & $\overline{\text { middle }}$ \\
\hline No. of pieces on board & 28 & 28 & 18 & 27 & 27 & 8 & 26 \\
\hline Time (minutes) & 29 & 16 & 10 & 15 & 15 & 20 & 7 \\
\hline No. of different moves & 115 & 66 & 51 & 66 & 38 & 68 & 51 \\
\hline Total No. of moves & 328 & 124 & 103 & 152 & 79 & 224 & 74 \\
\hline No. of episodes & 45 & 20 & 22 & 25 & 13 & 18 & 14 \\
\hline $\begin{array}{l}\text { No. of positive } \\
\text { evaluations }\end{array}$ & 39 & 14 & 11 & 9 & 22 & 18 & 7 \\
\hline $\begin{array}{l}\text { No. of negative } \\
\text { evaluations }\end{array}$ & 28 & 16 & 9 & 7 & 2 & 11 & 12 \\
\hline $\begin{array}{c}\text { No. of neutral } \\
\text { evaluations }\end{array}$ & 25 & 10 & 6 & 12 & 5 & 4 & 5 \\
\hline $\begin{array}{l}\text { Total No. of } \\
\text { evaluations }\end{array}$ & 92 & 40 & 26 & 28 & 29 & 33 & 24 \\
\hline Branchiness $^{a}$ & 2.04 & 2.00 & 1.13 & 1.12 & 2.23 & 1.83 & 1.64 \\
\hline Average depth ${ }^{b}$ & 3.57 & 3.08 & 3.96 & 5.43 & 2.72 & 6.79 & 3.22 \\
\hline$\%$ of different moves ${ }^{c}$ & 35 & 54 & 50 & 43 & 48 & 30 & 70 \\
\hline
\end{tabular}

a This is a measure of branching and is given by the ratio between the total number of evaluations and the total number of episodes.

${ }^{b}$ The ratio between the total number of moves considered and the total number of evaluations, where terminal evaluations have been substracted out, so as to distinguish the "process of branching" from the "termination" process.

- The ratio between the total number of different moves and the total number of moves considered. 
A, a portion of Sl's protocol, and the resulting Problem Behavior Graph are shown in the Appendix.

The protocols were analyzed by the two authors independently and we believe there is sufficient consistency to enable us to generalize. Wherever possible, we have followed Newell and Simon's methods of analysis. For instance, "Pawn threatens to take Knight now" is considered $\mathrm{P} \times \mathrm{N}^{\prime}$. When moves are inferred, but not explicitly mentioned they are put in parentheses; moves that are neither explicitly nor implicitly referred to are indicated by dotted lines.

Positive (+) and negative (-) evaluations ? judged from the $S$ 's assessinent of whether he has gained or lost mi rial or position at the end of a branch or episode. Thus, in Protocol A, I pisode 3, “. . . and I've caused the weakening of his Pawn structure. ." would be considered a positive evaluation $(+)$. We have defined the neutral evaluation $(0)$ to include evaluations that are unstated, equal or even, or undetermined. Thus, in Protocol A, Episode 18, "I might win a Pawn, but I'm certain to lose him back..." would be considered a neutral evaluation (0).

\section{Overall Comparisons Among Subjects}

Table 1 lists some comparative statistics for our $S \mathrm{~s}, \mathrm{~S} 1$ and $\mathrm{S2}$, and for S0, the $S$ of Newell and Simon. A quick comp rison of S0 and S1 on Position $\Lambda$ reveals some similarities. Figures 1 and 2 show that certain patterns differ not in the shape of the function but in the magnitude of the values. For example, as indicated in Tabl 1, with respect to the

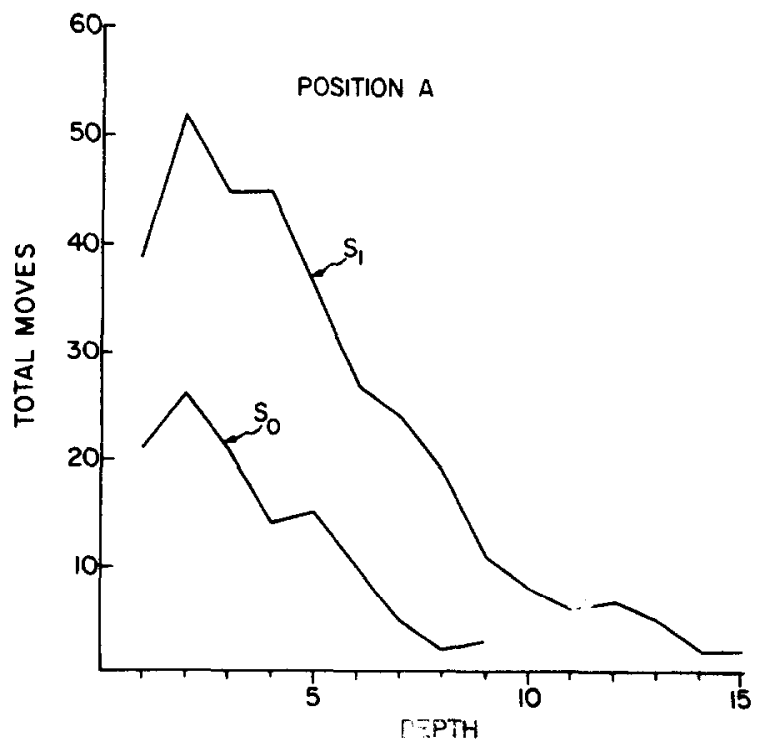

FIg. 1. The total number of moves as a function of depth on Position A. 


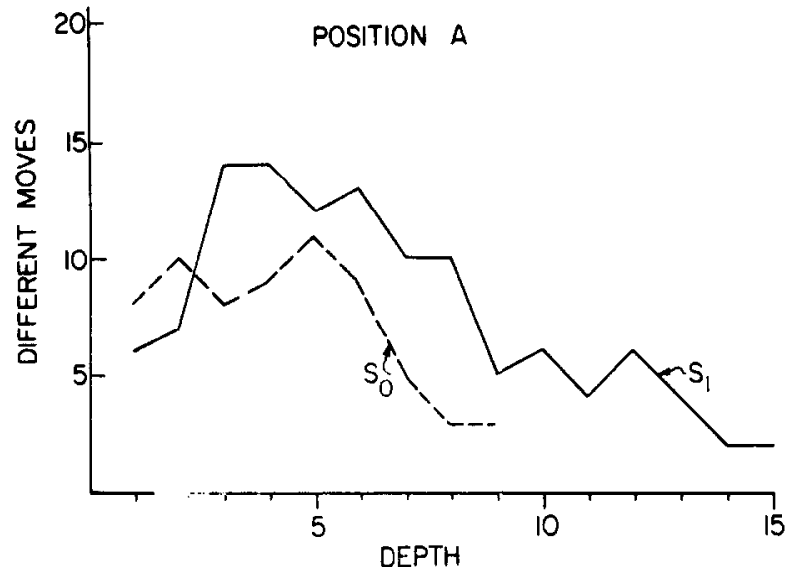

Frc. 2. The number of different moves as a function of depth on Position A.

measures of time, number of different moves, number of total moves, number of episodes, and number of evaluations, S1's values are consistently twice those of $S 0$. Since $S 1$ had available twice the time allowed $\mathrm{SO}$, it is reasc aable to suggest that these measures are indeed useful indicators of tuc course of the problem-solving process in chess.

In general, there $s \mathrm{em}$ to be no large systematic differences in the structure of strategie; (or problem-solving) between $S_{s}$ or between positions (middle, es 1 , or real game). Some interesting variations do occur, however. For xample, the large average depth of search in $\mathrm{E}$ (S1), an end game, 'night be explained by the very few chess pieces that had to be kept in memory during the analysis. Another case having a large average depth was $C(S 1)$. Here $S 1$ also had to cope with a large number of pieces, but the depth may be explained by the greater familiarity $S 1$ has with the real game position (C) that he had himself developed. Apart from this, there is no evidence to suggest that an artificially presented board position differs from the real game situation. Figure 3 compares the gross data output (total moves versus depth) of $S 1$ and $S 2$.

In general, the tim. constraints seemed to cause no large systematic differences in Sl's or rall problem-solving behavior, although several points can be made. ( 1 e conclusion is that the more pressing the time limits, the larger the trcentage of different moves S1 scans. Table 2 shows a clear negativi relationship between the percentage of different moves (different mov:;/total moves) considered and the time taken in deciding upon a move (the regression slope is significant (t-test) at $p<.025)$. This relationship suggests that longer time limits allow the $S$ to be more repetitious (thorough?) in his analysis. Another interesting measure is that of the "processing rate" (moves/minute) of each $S$ on a 


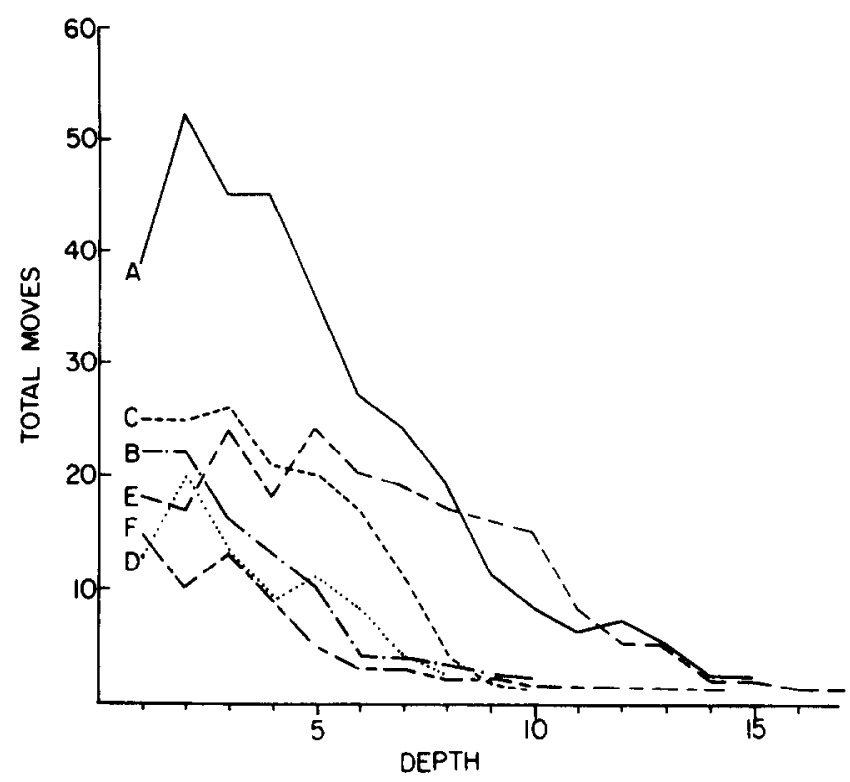

Fic. 3. The total number of moves as a function of depth on Positions A-F ( using $S 1$ and S2).

particular board position (Fig. 4). This is linear for $S 1$ at about 10 moves per minute (regression slope significant ( $t$-test) at $p<.01$ ). A measure related to the above points is the rate of scanning search, which, for $S 1$, is about four different moves per minute. There are not enough data here, however, to state that each $S$ has a characteristic "processing rate."

In summary, although we have shown some characteristics of human chess problem-solving here, we have not found any large differences in problem-solving behavior between $S$ s and positions that are attributable to the changes in conditions introduced by the experimenters.

The Model of Newell and Simon (1965)

Let us now apply the Newell and Simon model to our data. There are three components to the model that are relevant to this discussion: the

TABLE 2

Decision Times and Percent Use of Different Moves

\begin{tabular}{|c|c|c|c|c|c|c|c|}
\hline Position-Subject & $\mathrm{F}(\boldsymbol{S} 1)$ & $\underline{B(S 1)}$ & $C(S 1)$ & $\mathrm{D}(\mathrm{S} 2)$ & $\mathrm{A}(\mathrm{SO})$ & $\underline{\mathbf{E}(S 1)}$ & $\mathbf{A}(\mathbf{S} 1)$ \\
\hline $\begin{array}{l}\text { Time taken for } \\
\text { decision }\end{array}$ & 7 & 10 & 15 & 15 & 16 & 20 & 29 \\
\hline$\%$ different moves & 70 & 50 & 43 & 48 & 54 & 30 & 3.5 \\
\hline
\end{tabular}




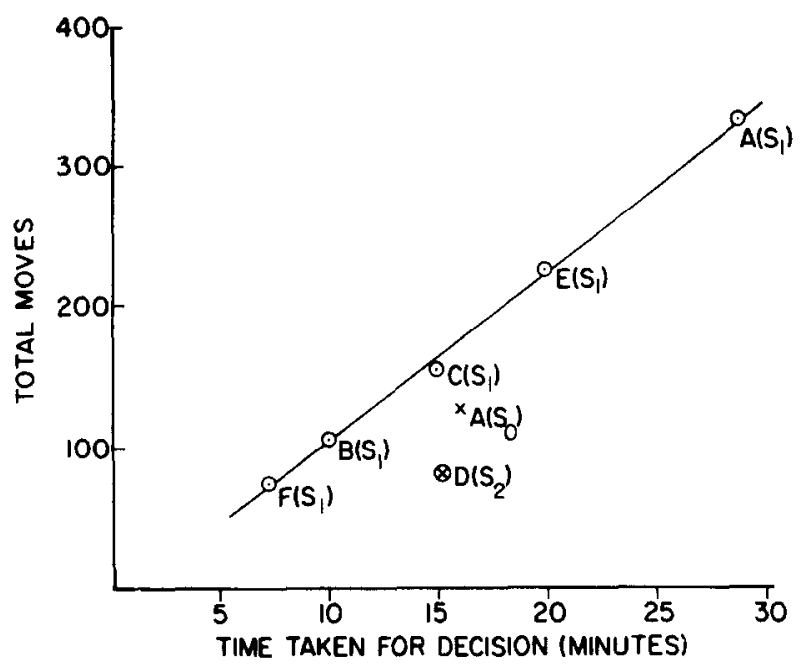

Fic. 4. The total number of moves as a function of protocol time taken for decision as to choice of move on Positions A-F. Regression line through S1 only.

first deals with the specific choice of moves; and the others deal with how the $S$ decides among classes of moves (e.g., base move or line move), and thus determines the structure of his problem-solving search.

Generation of situation-response moves (GSRM). The first component is a system of situation-response generators (GSRM) which answers the question "What particular move will $S$ choose next?" Newell and Simon suggest that $S$ recognizes certain "functions to be performed"- that $S$ has "an available collection of fixed responses to specific situations" (1965, p. 50). For example, among their situation-response rules they list: "man captures last move-recapture with no apparent loss," and "man attacked-counter-attack of equal value." We accept this idea and we shall use it later in our model.

Modified progressive-deepening. The second part of this theory, the "Modified" Progressive-Deepening Search Strategy" (MPD), was an attempt to specify the hypothetical structure of S0's problem-solving behavior as displayed in the analysis of episode branching. MPD does not attempt to reproduce or explain the actual mental processes of S0, but they hope that the "hypothesized organization is sufficient to reproduce the $S$ 's observed behavior." In general, this type of structural modeling is the first step toward computer simulation, which was one of the aims of Newell and Simon.

MPD is given as follows.

" The "Modified" refers to a broadening of their originally hypothesized "Progressive-Deepening Search Strategy." 
In considering the base position:

-Either an old base move (i.e., one from the base position) is reconsidered, or a single new base move is generated and considered.

-A summary is kept of the state of analysis of each base move.

In considering a position, $\mathrm{X}$, other than the base position:

-The state of analysis of the base move leading to $X$ is available.

-If $X$ is static, return to the base position.

-If $\mathrm{X}$ is dynamic, generate a set of moves from $\mathrm{X}$. Consider each in turn, but only statically. Select one of these moves and consider it dynamically.

The terms "static" and "dynamic" refer to the evaluation state of each move. A move that is "static" is capable of being evaluated. A move is "dynamic" if it leaves matters in a state in which evaluation is impossiblc. As Newell and Simon say of MPD, "This strategy will produce a sequence of explorations, each starting from the base position, but with the tree of each exploration resembling a skinny Christmas tree: the tree would have a main trunk, and at each node there would be a tuft containing a number of branches, each one more deep" [p. 40].

Rules for episode sequence. In their analysis and discussion of the episodes into which their protocol was divided, Newell and Simon suggested six rules for generating a sequence of episodes, which could hypothetically crcate a FBG like that they show for S0. These Rules for Episode Sequence (RES), a third component of their model, sought to predict which base move would be selected for the next episode and what context governed the exploration of subsequent episodes of the same basis. The following is a listing of RES:

RES 1: The analysis of each base move is independent of the analysis of other base moves, except that it can be interrupted by other activity. That is, each episode in the analysis of a base move is determined only by the results of the prior episodes of that base move.

RES 2: The first episode of the base move employs normal moves and subsequent episodes utilize increasingly unusual moves.

RES 3: If the evaluation of an episode gives a favorable result, then an analysis of its base move is continued; if the evaluation is unfavorable, a different base move is analyzed.

RES 4: When exploring, moves for the opponent may be considered that are favorable to self (in order to place an upper bound on the possibilities).

RES 5: The analysis of a base move will be interrupted to pursue other moves, discovered during the episode, that seem to have merit either for self or for the opponent. 
RES 6: Before a base move is finally chosen, a check is made for other alternative base moves.

After analysis of the 25 episodes (or 20 episodes, using our definition of episode) in their $S$ 's protocol, Newell and Simon concluded that "there is substantial evidence that the $S$ is observing the first three rules; and more limited evidence for the remaining three" [p. 43].

The data for S1 and S2 generally are consistent with Newell and Simon's findings, except in the case of RES 3. In general, where the RES were applicable, they were confirmed. However, RES 4-6 had relatively few applications in our data. RES 6 did not apply in any of the five protocols of $S 1$, although it did apply to the one protocol of S2-suggesting that it is an individual, non-general, search strategy.

RES 3, which had the most application (90\%) and the least conformations (58\%) among the RES, is worth considering further. Table 3 lists the number of cases in our data in which RES 3 was applicable, confirmed or disconfirmed. Newell and Simon summarize RES 3 by saying: "Stay with a winner, switch off a loser." That is, base moves giving positive evaluations will continue to be explored until a negative evaluation is reached, where a new base move will be explored. RES 3 was highly confirmed by $S 0$ and $S 2$, and poorly confirmed by $S 1$, which again may indicate that the Ss used different search rules, as with RES 6 .

Table 4 is a detailed analysis of the disconfirmations of RES 3 , broken down by protocol and nature of disconfirmation. Also shown in Table 4 is the percentage of episodes starting from the "most favored" (most frequently analyzed) base move in each protocol with regard to S1. A larger proportion of disconfirmations of Type 2, a negative evaluation followed by the same base move, was associated with a high concentration on the analysis of a single base move (e.g., Positions A, C, and E). Type I disconfirmations, switching from a positive evaluation to a new base move, had a low concentration of the analysis of a single base move (e.g., $B$ and F). A reasonable explanation for the Type 2 dis-

TABLE 3

Summary of RES 3 Applications

\begin{tabular}{|c|c|c|c|c|c|c|c|c|}
\hline & \multicolumn{8}{|c|}{ Protocols } \\
\hline & $\underline{\mathrm{A}(\boldsymbol{S} 1)}$ & $\underline{\mathrm{B}(\mathrm{S} 1)}$ & $\mathrm{C}(\mathrm{S1})$ & $\underline{\mathrm{E}(S 1)}$ & $\mathbf{F}(S 1)$ & $\begin{array}{c}\text { Total } \\
S 1 \\
\end{array}$ & $\mathbf{A}(S 0)$ & $\mathrm{D}(S 2)$ \\
\hline Confirmations & $\overline{26}$ & $\overline{8}$ & $\overline{12}$ & $\overline{12}$ & 8 & 66 & $\overline{16}$ & $\overline{11}$ \\
\hline Disconfirmations & 15 & 9 & 11 & 5 & 7 & 47 & 2 & 1 \\
\hline Non-applications & 4 & 5 & 2 & 1 & 0 & 12 & 7 & 1 \\
\hline Total & 45 & 22 & 25 & 18 & 15 & 125 & 25 & 13 \\
\hline Percent confirmations & 63 & 45 & 52 & 70 & $5 \overline{5}$ & 58 & 90 & 92 \\
\hline Percent applications & 93 & 77 & 92 & 94 & 100 & 90 & 72 & 92 \\
\hline
\end{tabular}


TABLE 4

Types of Disconfirmations-RES 3

\begin{tabular}{|c|c|c|c|c|}
\hline Protocol & $\begin{array}{c}\text { A positive } \\
\text { evaluation, } \\
\text { followed by a } \\
\text { new base } \\
\left.\text { move ( } T_{y} \text { pe } 1\right)\end{array}$ & $\begin{array}{l}\text { A negative } \\
\text { evaluation, } \\
\text { followed by } \\
\text { same base } \\
\text { move (Type 2) }\end{array}$ & $\begin{array}{l}\text { Total discon- } \\
\text { firmations }\end{array}$ & $\begin{array}{c}\% \text { use of } \\
\text { "most fa- } \\
\text { vored" base } \\
\text { move }\end{array}$ \\
\hline$\overline{\mathrm{A}(S 1)}$ & - & 15 & 15 & 68 \\
\hline $\mathrm{B}(S 1)$ & 5 & 4 & 9 & 36 \\
\hline $\mathrm{C}\left(\mathrm{S}_{1}\right)$ & 1 & 10 & 11 & 60 \\
\hline $\mathrm{E}(S 1)$ & - & 5 & 5 & 100 \\
\hline$\Gamma(S 1)$ & 3 & 4 & 7 & 25 \\
\hline Total (S1) & 9 & 38 & 47 & 57 \\
\hline $\mathrm{A}(S 0)$ & - & 2 & 2 & $57^{a}$ \\
\hline $\mathrm{D}(S 2)$ & - & 1 & 1 & 77 \\
\hline
\end{tabular}

a In calculating, the number of episodes has been adjusted from 25 to 20 to make comparisons possible.

confirmations may be that when $S 1$ analyzed a base move and found that it yielded primarily positive results early in the protocol, he was encouraged to explore this line further. As the positive evaluations continued, S1 built up an "investment" of time and effort (and memory storage) in this base move and thus was reluctant to abandon it for another base move when negative evaluations showed up later in the analysis. This seems to explain Protocols $A$ and $E$ fairly well. Episodes 3-6 and 8-10 of Protocol A, and 1-4 and 6-9 of Protocol E, began with the most frequently explored base move and ended with positive evaluations. Later in the protocols, negative evaluations often were followed by the same base move (e.g., Protocol A, Episodes 14, 16, and 17).

However, this explanation does not fit Protocol C. In this case the favored base move continued to be analyzed despite neutral and negative evaluations from the beginning. Furthermore, this persistence led $S 1$ to choose an exceedingly bad move. To find an explanation, we must note again that Position $\mathrm{C}$ was developed from the beginning by $\mathrm{S} 1$, and thus he had not only prior knowledge, but perhaps also a prior "investment" in a certain move strategy. For even in the first episode of Protocol C, SI said: "So the most natural move is Pawn to King 5. . .." In sum, it appears that S1 made Type 2 disconfirmations when he had some sort of cognitive investment in a particular move, either by previous development, or during problem analysis.

A reasonable explanation for the Type 1 disconfirmations abundant in Protocols $\mathrm{B}$ and $\mathrm{F}$ is the fact that $\mathrm{B}$ and $\mathrm{F}$ had the most constrained time limits ( 10 and 7 minutes, respectively). As we noted before in the discussion of the effects of time limits, the more constrained the time, the more 
widely S1 searched. Thus, Type 1 disconfirmations-“"switching from a winner" - would be necessary for an appropriately wide search.

\section{An Alternative Theory of Move and Episode Generation (MEG)}

We have discussed the rules in RES, particularly RES 3, and have found them less than complete for describing the behavior of our $\mathrm{Ss}$, and particularly $S 1$ (for whom we have the most data). We have suggested several explanations for the differences, but we have not yet offered any hypotheses that would explain Sl's behavior in any detail. This section presents a set of rules that encompasses some of the ideas in MPD and RES, but explains Sl's behavior to a greater extent than the Newell and Simon model, and is also applicable to S0 and S2.

With MPD and RES, Newell and Simon propose an integrated description of the behavior of their $S$. The following is a set of rules for Move and Episode Generation (MEG) that determines the episode and branching structure, and thus describes a part of the decision behavior of our $S$.

MEG 1: (a) If the evaluation is negative ( - ), the first new move in the next branch or episode, i.e., a new line move, will be made by the $S$.

(b) If the evaluation is positive $(+)$, the first new move in the next branch or episode, i.e., a new line move, will be made by the opponent.

MEG 2: A generated new move will replace the last move made by the opponent or $S$ in accordance with MEG 1 . In a few cases, the second-to-last move will be replaced, rather than the last move.

MEG 3: If the evaluation is negative (-) and all plausible line moves have been considered, the new move generated will be a new base move.

MEG 4: If the evaluation is positive $(+)$ and the $S$ has a time constraint or limit, he may switch to a new base move.

MEG 5: If the evaluation is positive $(+)$ and all plausible line moves have been considered, the present base move will be chosen by the $S$.

MEG 1 says: "That is not good for me $(-)$, what other move can I make?" or "That is good for me $(+)$, but what other moves can my opponent make?" This rule forms the basis of new move generation not only within episode branching, but also in the choice of branch moves. RES 3 says, "Switch from a loser, stay with a winner." Thus, MEG 1 is not contradictory to RES with regard to new base moves. However. 
MEG 1 goes further than RES 3 with regard to non-base move generation. MEG 1 says that branching may occur even if preceded by a negative evaluation. Thus the $S$ is not "switching from a loser," but is attempting to rectify a bad situation within the same base move. MEG 1 is illustrated in Fig. 5; one can see that this rule allows for two types of new move generation: base moves and branch (or line) moves. A further specification to determine which type this new move will be is contained in MEG 2-4.

MEG 2 is described empirically in Table 5, which shows that new moves occur in "primary placement" with 78\% occurrence, and in "secondary placement" with $12 \%$ occurrence. An illustration of the structure that MEG 2 imposes on MEG 1 can be seen in Fig. 5. Two methods of branching are shown. "Primary placement" of branching stands for the event when the new move replaces the last move made by the opponent or S (e.g., $\zeta^{\prime}$ replaces $\delta^{\prime}$ ). "Secondary placement" of branching stands for the event when the second-to-last move is replaced by the new move (e.g., $\theta^{\prime}$ replaces $\delta^{\prime}$ ). For example, in Position A, Episode 29, S1 says:

TABLE ;

Statistical Data on MEG 1 and MEG 2

\begin{tabular}{|c|c|c|c|c|c|c|c|c|}
\hline & \multicolumn{8}{|c|}{ Protocols } \\
\hline & $\mathrm{A}(S 1)$ & $\mathrm{A}(\mathrm{S} 0)$ & $\mathrm{B}(S 1)$ & $\mathrm{C}(S 1)$ & $\underline{\mathrm{D}(S 2)}$ & $\mathrm{E}(S 1)$ & $\mathbf{F}(S 1)$ & Total \\
\hline \multicolumn{9}{|l|}{$M E \because ; 1$} \\
\hline No evaluations & 25 & 10 & 6 & 12 & 5 & 4 & 5 & 67 \\
\hline Total evaluations & 92 & 40 & 26 & 28 & 29 & 33 & 23 & 271 \\
\hline Confirmations & 66 & 27 & 20 & 14 & 23 & 28 & 17 & 195 \\
\hline Jisconfirmations & 1 & 3 & 0 & 2 & 1 & 1 & 1 & 9 \\
\hline Applications & 67 & 30 & 20 & 16 & 24 & 29 & 18 & 204 \\
\hline$\sigma_{c}$ confirmations & 98 & 90 & 100 & 87 & 95 & 96 & 94 & $9 \bar{j}$ \\
\hline \multicolumn{9}{|l|}{$M E G \quad 2$} \\
\hline $\begin{array}{l}\text { Branches with no } \\
\text { evaluation }\end{array}$ & 15 & 9 & 1 & 1 & 1 & 1 & 2 & 30 \\
\hline $\begin{array}{l}\text { Total No. of } \\
\text { branches }\end{array}$ & 50 & 16 & 4 & 3 & 17 & 15 & 8 & 113 \\
\hline $\begin{array}{l}\text { Primary con- } \\
\text { firmations }\end{array}$ & 31 & 4 & 2 & 1 & 14 & 9 & 4 & 65 \\
\hline $\begin{array}{l}\text { Secondary confir- } \\
\text { mations }\end{array}$ & 4 & 1 & 1 & 0 & 2 & 1 & 1 & 10 \\
\hline J)isconfirmations & 0 & 2 & 0 & 1 & 0 & 4 & 1 & 8 \\
\hline Applications & 35 & 7 & 3 & 2 & 16 & 14 & 6 & 83 \\
\hline$\%$ confirmations & 100 & 71 & 100 & 50 & 100 & 71 & 83 & 90 \\
\hline $\begin{array}{l}\text { Average branch } \\
\text { length }\end{array}$ & 1.8 & 1.5 & 2.0 & 1.0 & 1.6 & 2.2 & 1.9 & 1.6 \\
\hline
\end{tabular}


So if Queen takes Pawn; say, Rook to either at the moment; and Queen takes the other Pawn; I now have Knight to Bishop 4, hitting the Queen. . . . No, mayle it would have been better to go to Rook to Knight 1 , and cut off his squares on the Knight file. ...

MEG 3 presents a new word: "plausible." "Plausible line moves" are not difficult to conceptualize if they are seen in terms of the normal-to-

\section{RES 3 vs MEG 1}

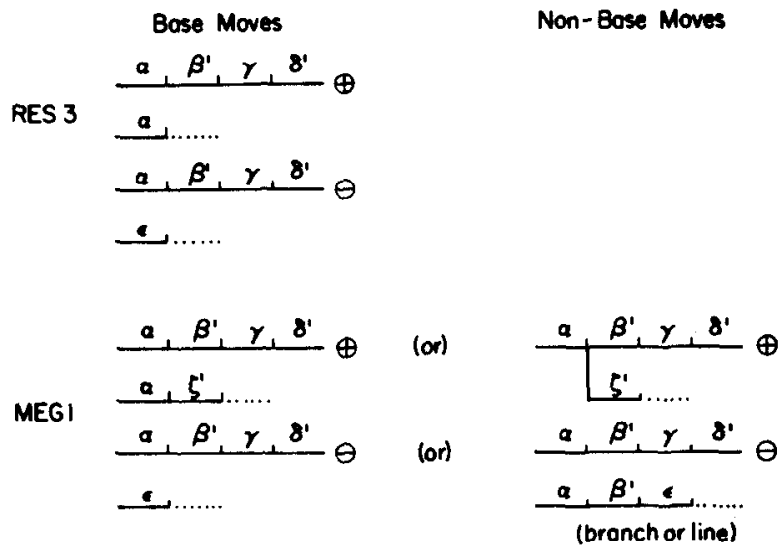

PLACEMENT OF NEW MOVE (MEG 2)

I. "Primary Place" of Branching:
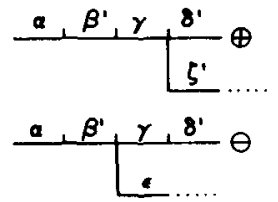

(end in block)
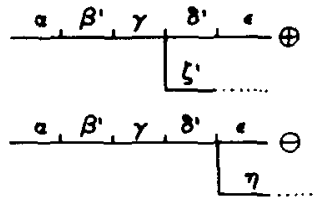

(end in while)

2. "Secondary Ploce" of Bronching:
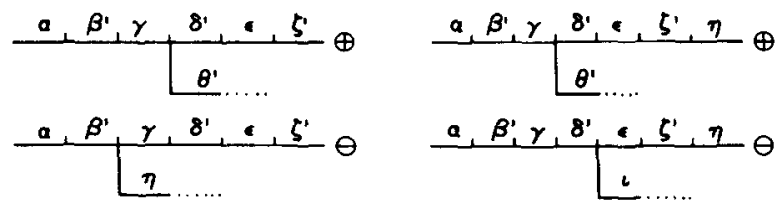

Frg. 5. A comparison of RES 3 with MEG 1; and an indication where branching will take place, in accordance with MEG 2. 
unusual sequence of moves seen in RES 2 earlier. Plausible moves appear in this sequence; and "all plausible line moves have been considered" when the $S$ has reached his subjective limit of what is "unusual." He will then switch to a new base move from his normal-to-unusual sequence of base moves. The concept of "plausible moves" here is similar to the "plausible move generators" of computer programs (Newell et al., 1958; Greenblatt et al., 1967).

MEG 4 is simply a derivative from the earlier discussion of Type 1 disconfirmations of RES 3 . It indicates that the $S$ may not "stay with a winner" (a positive evaluation) if he desires to scan the possibilities quickly (a wide search) under what the $S$ feels is a time constraint. This type of quick-scan strategy is found in human decision-making tasks under time constraints. Yet, even this wide search for alternatives will not prevent the $S$ from returning to an analysis of previously favored base moves. MEG 5 is intuitively reasonable, and perhaps only an extreme time limit would prevent the $S$ from considering all his plausible line moves.

\section{Analysis and Discussion}

MEG 1 and 2 will be analyzed with the tabulated data of Protocols A to $F$ listed earlier in Table 5. This thcory, of coursc, relies upon the existence of an evaluation, but the fact that branches with no evaluation (25\% occurrence) appear identical to the evaluated branches indicates that often some type of evaluation may be implicit, but not verbalized by the $S$. The verification of MEG is made somewhat easier than that of RES because MEG was for the most part empirically derived. We see that MEG 1 is confirmed at a consistency over $90 \%$ in almost every protocol ( 195 confirmations out of 204 applications); this is a high figure when compared to RES. MEG 2, the rule for placement, has $90 \%$ total confirmations ( 75 out of 83 applications).

As mentioned earlier, the criterion for judging "plausible" was the same as the normal-to-unusual sequence of RES 2. MEG 3 is an adapted part of RES 3 which says: "If the evaluation is unfavorable, a different base move is analyzed." Given that MEG 4 and MEG 5 do not have priority, we can find several ex:mples of MEG 3. A clcar cxample is Position B, Episode 19, where $S 1$ is finally going to give up developing B'-Q2 (i.e., all plausible line moves have been considered). He said: "And, all in all, that seems to be a good line for him. So I think I'm going to give up on Bishop to Queen 2. . ." With regard to the negative evaluation leading to a new base move, this follows the normal MEG 1 pattern-namely, "what other move can I make?" Therefore, discon- 
firmations of this are rare, and are included in the MEG I disconfirmations.

The rule MEG 4 is "weak" in that it is not widely applicable in our protocols-although, for example, five applications occur in Protocol B. An example from Protocol B, Episode 1, states: "A Rook to Knight 1 move is possible, attacking his Knight Pawn; force a possible weakening in it, but I'm looking for something sharper here, because I'm down a bit of material, and I'd like to see if I could win something back immediately. . . ." It seems likely that with more constrained time limits than 7 or 10 minutes, there would be even more forced scanning of available moves.

MEG 5 is relatively easy to test. If the last evaluation is positive and the $S$ feels that he has considered all plausible line moves (as in MEG 3 ), he will choose that present base move. For example, in Protocol B, Episode 22, SI said: "guess I can't ask for much better . . . I really don't have much else in this position," and chose the present base move as final. Thus, MEG 5 was confirmed in Protocols A, B, C, D, and F. The exception was Protocol E where S1 continued his first and only choice, in the end trying more unusual alternatives to improve upon it.

On the whole, MEG appears to describe Sl's behavior quite well, and

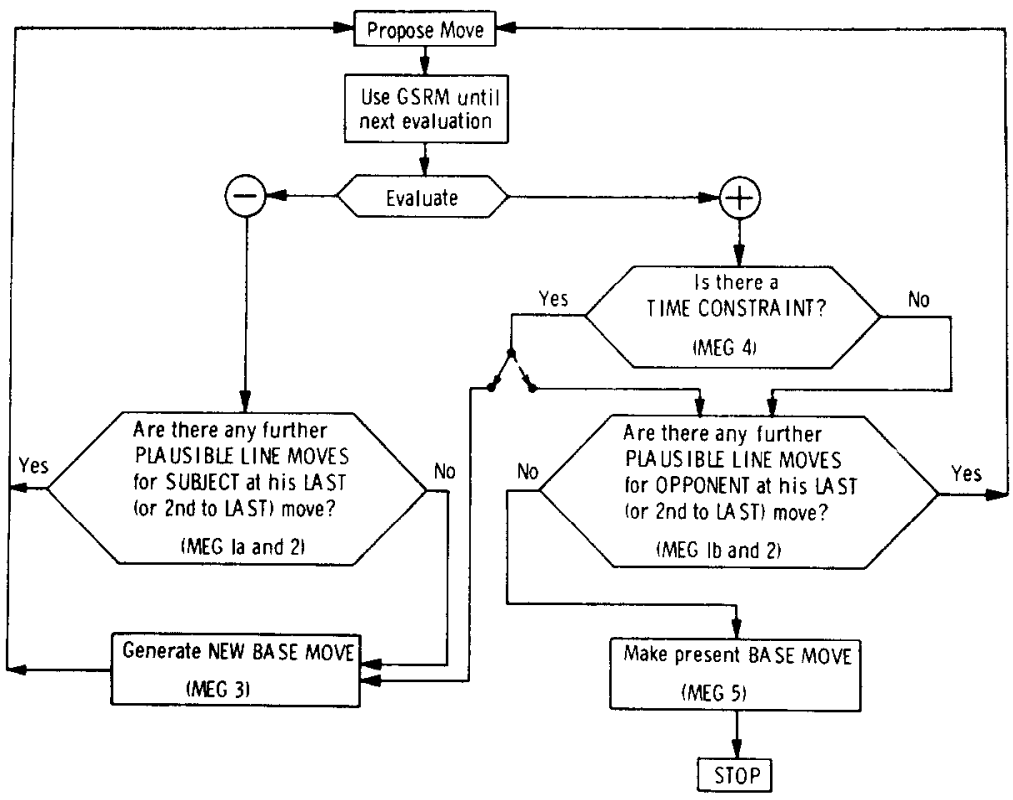

Fig. 6. A How chart of the rules for Move and Episode Generation (MEG I-5). The "branch point" with the dotted and solid arrows (following MFG 4) is to indicate uncertainty in that branch. 
does almost as well with S0 and S2. The data shown in Table 5 indicate a strong confirmation of MEG 1 and MEG 2. MEG 3-5 have fewer applications, but the data generally seem confirmatory.

The flow chart in Fig. 6 shows the MEG rules in a schematic form (recall that GSRM stands for the Newell-Simon proposal accounting for the generation of specific moves).

Search strategies. Previously we briefly discussed two strategies and the structure of episode branching that they produce: MPD and MEG. The general features of MPD are the existence of episodes with a return to a base move, and "single level tufts at each branching node." Although the first part is in no conflict with MEG, the second part (single move tufts) causes some difficulty. Newell and Simon suggest that the $S$ comes

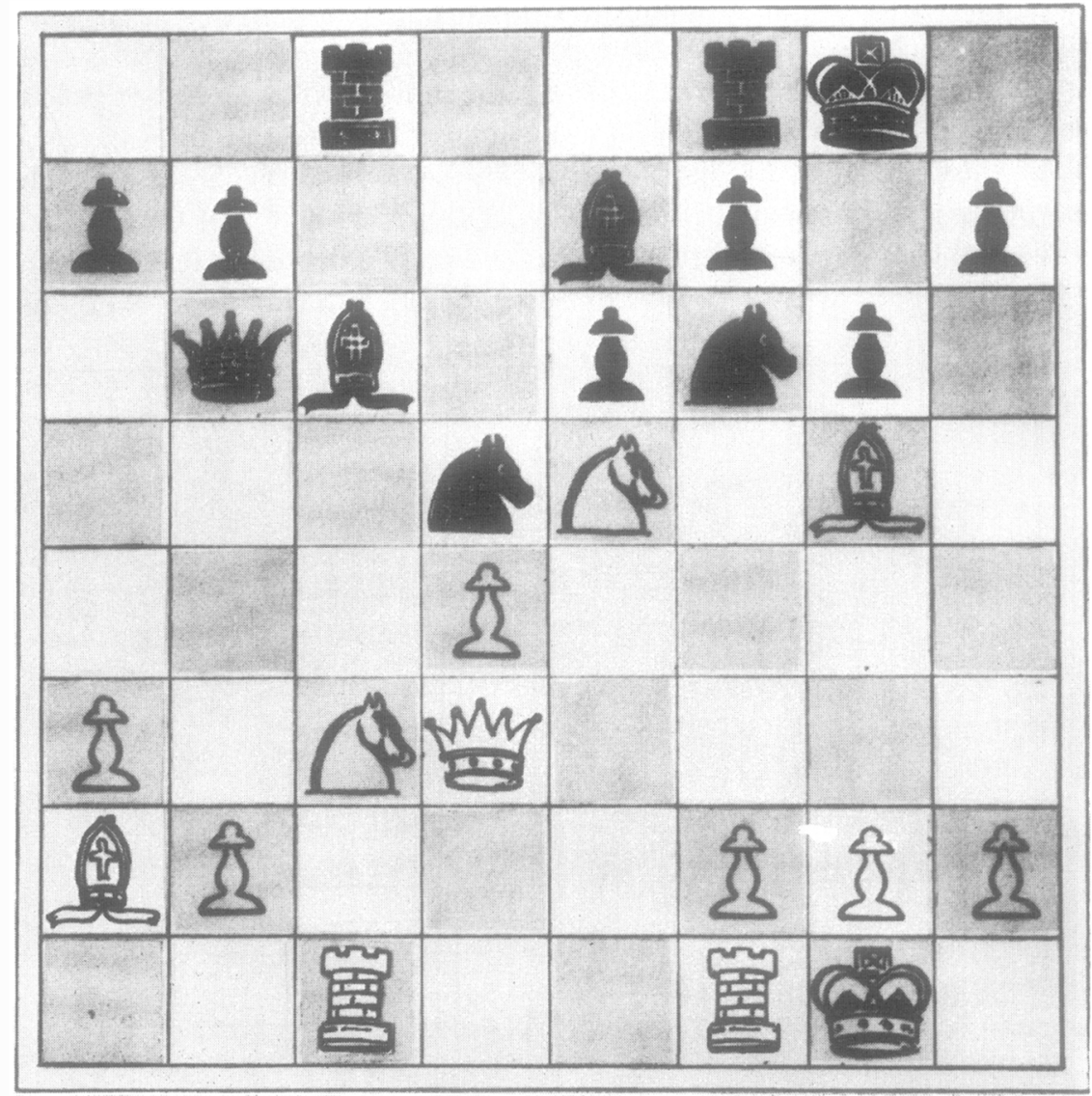

Fic: 7. Position A (de Groot, 1965). Used by the authors with S1, and previously by Newell and Simon (1965) with SO. It is white's move; the choice made by $\mathrm{S} 1$ was $\mathrm{N} \times \mathrm{N}^{\prime}$. (Note: The black pieces are "primed" in the written text.) 
to a certain (dynamic) point and generates a series of alternatives, each one of which is considered, and selects one for deeper searching. The basis for the application of MPD to their $S$ apparently rests on the word "or." For example, in S0, Protocol A, Episode 21, their S says: "It can, of course, move to Rook 4-can move to Rook 4 or it can retreat to Bishop 2 or Queen 1. . ." Thus, we see that the word "or" (possibly "and" in some cases) is actually the only way for a $S$ to consider alternatives and then pick one; that is, this manner of speaking is necessary for MPD. With respect to single tuft branching, S0 seemed to follow MPD with some consistency (11 out of 15 instances of multiple branching), while $S 1$ did so only 16 out of a possible 35 instances-or less than $50 \%$ of the time.

If MPD is applied to $S 1$, serious problems arise. Single branches occur in only half the cases of branching for S1. There are many episodes that have a two move branch and several with multiple branching with each branch two or more moves long (e.g., Position A, Episode 34; Position $\mathrm{E}$, Episode 10). Thus, for MPD to work here, the $S$ would sometimes have to generate alternatives that were two moves deep and then choose one to consider more deeply. The net effect of this would be a doubling (or more) of the memory that is needed to consider the alternatives. That is, each alternative has an average of two moves compared to the original MPD theory of a single move. It seems, then, that S1 shows little indication of using MPD search strategy.

\section{REFERENCES}

BAYLOR, G. W. Report on a matings combination program. S.D.C. Paper No. SP-2150, Systems Development Corporation, Santa Monica, CA, 1965.

BAYLOR, G. W., \& Simon, H. A. A chess matings combinations program. In Proceedings of the Spring Joint Computer Conference, Washington, D. C.: Spartan, 1966.

Bernstein, A., \& Roberts, M. De V. Computer vs. chess player. Scientific American, 1958, 198, 96-105.

De Groot, A. D. Thought and choice in chess. The Hague: Mouton, 1965.

Fine, R. The middle game in chess. New York: David McKay, 1952.

Greenblatt, R. D., Eastland, D. A., \& Crocker, S. D. The Greenblatt chess program. In Proceedings of the Fall Joint Computer Conference. Washington, D. C.: Thompson, 1967. Pp. 801-810.

Newell, A. On the analysis of human problem solving protocols. Calcul et Formulisation dans les Sciences de lHomme, Editions du Centre National de la Recherche Scientifique, Paris, 1968. Pp. 145-185.

Newell, A., Shaw, J. C., \& Simon, H. A. Chess-playing programs and the problem of complexity. IBM Journal of Research and Development, 1958, 2, 320-335.

Newell, A., Shaw, J. C., \& Srmon, H. A. Empirical explorations with the logic 
theory machine: A case in heuristics. In E. A. Feigenbaum and J. Feldman (Eds.), Computers and thought. New York: McGraw-Hill, 1963. Pp. 109-133. Newell, $\Lambda$., \& Simon, H. $\Lambda$. GPS, a program that stimulates thought. In E. A. Feigenbaum and J. Feldman (Eds.), Computers and thought. New York: McGraw-Hill, 1963. Pp. 279-296.

Newell, A., \& Simon, H. A. An example of human chess play in the light of chess playing programs. In N. Weiner and J. P. Schade (Eds.), Progress in biocybernetics. Amsterdam: Elsevier, 1965. Pp. 19-75.

Scurrah, M. J., \& Wagner, D. A. Cognitive model of problem-solving in chess. Science, 1970, 169, 209-211.

Shannon, C. E. Programming a digital computer for playing chess. The Philosophical Magazine, March, 1950, 356-375.

Simon, H. A., \& Simon, P. A. Trial and error search in solving difficult problems: Evidence from the game of chess. Behavioral Science, October, 1962, 425-429.

\section{APPENDIX: POSITION A}

\section{Protocol of Subject S1}

Well, it looks like White has the initiative Black's King side is weakened with its Pawn on King Knight 3 its got a centralized Knight at King 5 . . . strong position it's Queen, Bishop seem to be ready to line up. And ... uh . . . it's a complicated position as ... uh . . . obviously there's not going to be any sacrifice . . . I say obviously because Black has a relatively well developed position with good control of the strong points. So it looks like it's, uh, positional plan here I would look for any immediate tactical shots. Perhaps if we actually play on the Queen side rather than on the King side here because, uh, we actually have the Queen Bishop file here ready for use and

Episode 1 the Knight on King 5 hitting the Bishop there, Queen Bishop 3, if we could force a weakening in the Pawn structure so we could recapture with a Pawn and we'd have a target on the Bishop file but at the moment that's not quite feasible because it's too well protected.

It's not (attacked) often enough This Pawn Queen 4 is also an alternative but that also seems to be attacked very well

Episode 2 So off hand it's not too clear to me what's a strong move here 
And now I might try something different

like taking off a Knight here

and remove some of his defense

and see how that would affect things

so if I look at Bishop takes Knight,

he has a couple of ways of recapturing

If he takes back with the Knight

and then [I] take his Bishop-

recaptures with his Knight-

and I've decentralized his position

of course I've made too many exchanges.

Not too good

unless I can get something definitely out of it

But I could now play Knight into King 4

and that would uh enable me to uh put pressure

on his weak black squares

which is also the [weaker]

because his black squares Bishop has been cut off

But this Bishop is controlling King 4 unfortunately

so you've clipped the Knight right off

and I'm back where I started from

It's very difficult...

Let's see... .

It's very hard to think of something here, I uh ...

Episode 3 Try another line.

Seems to be the best opportunity.

Immediate capture of Qucen 5 again

Again, if we go Knight takes Knight

Well, hut I gness last time

my Bishop takes Knight.

Let's continue on that line

and sce if we can exhaust that possibility

again

Now, now let's see

Now let Knight takes Knight,

we'll try that again and see if anything else comes up

Knight takes Knight

he plays Knight takes Knight

now I can't play Bishop takes Knight,

leaving that Bishop in the air

Now, if he goes Bishop takes Bishop 
I can put a Bishop takes Bishop and I've caused the weakening of his Pawn structure on the Queen side which was one of my original ideas because uh he has to recapture with the Pawn and Knight guarding the Bishop

Episode 4 So let's look at that again and Knight takes Knight and he goes Knight takes Knight

I go Bishop takes Knight Now he cannot play Bishop takes Bishop But Bishop takes King ... Queen Bishop So he must rccapture this Bishop here But if he recaptures this Bishop here his other Bishop I take off next move So this seems to be fairly promising

Episode 5 Try that one more time

Knight takes Knight Knight takes Knight Bishop takes Knight Now his Bishop on King 2 is hanging as he cannot recapture my Bishop on Queen 5 so he must take (back to the old variation) I then take over here on Bishop 6 he recaptures and uh I have achieved my objective of weakening his Pawn structure

Episode 6 Now uh let's see...

His only chance to veer from that line would be on a first move of the alternative So again, Knight takes Knight He does not have to play Knight takes Knight leaving his Bishop open to attack. He has 2 alternatives, both Bishop takes Knight and Pawn takes Knight Uh, I'll look at Bishop takes Knight first because Pawn takes Knight because it would probably be favorable to me because it blocks the scope of his Bishop 
and in general would be a move he wouldn't want to make, I don't think

Episode 7 So let's look at what would probably be his better move so play Knight takes Knight

He plays Bishop takes Knight

Now an idea here is instead of immediately recapturing Bishop takes Bishop

because that allows him to safely play Knight

takes Bishop

is to now interpolate Bishop takes Knight

The idea behind that is if he now normally recaptures

I can play Bishop takes Bishop

and then he cannot recapture the Knight

it having been recaptured

and he must play Pawn takes Bishop

Trying to weaken his Pawn structure

but it wouldn't be weakened I guess

because his Bishop's off the board

so I haven't blocked the scope of his Bishop

which was the idea,

but let's look at it anyway

Episode 8 Knight takes Knight

Bishop takes Knight

Bishop takes Knight

and if he just takes the normal move,

Bishop takes Bishop

I play Bishop takes Bishop

plays Pawn takes Bishop

and the position seems to be approximately equal

except I just noticed that I have a fork on

Queen 7 with my Knight

which wins material

and therefore this alternative is ruled out for him

which is another good sign ...

A little light in the darkness here

Episode 9 So to look at that again

Knight takes Knight

Bishop takes Knight

Bishop takes Knight ... 


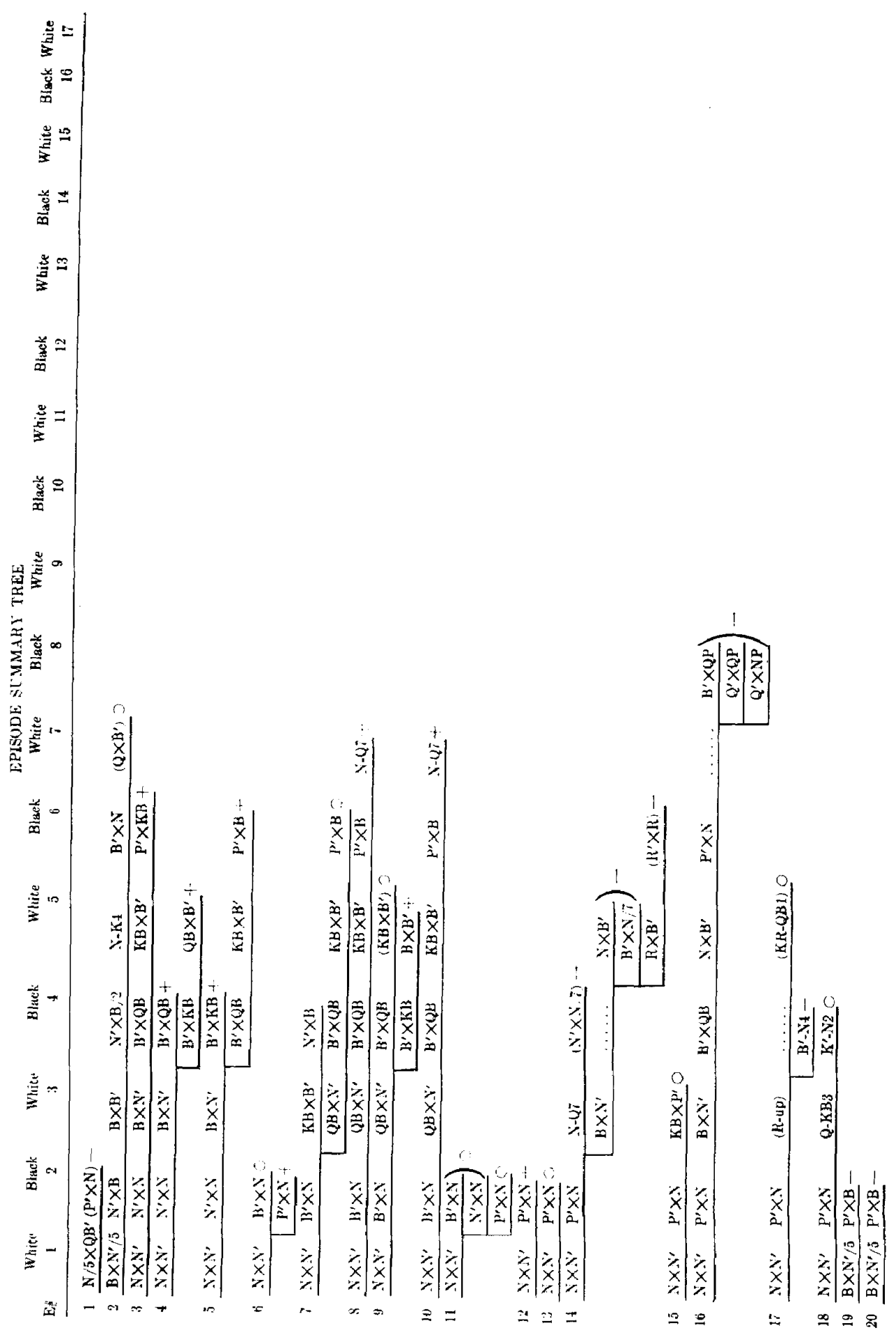




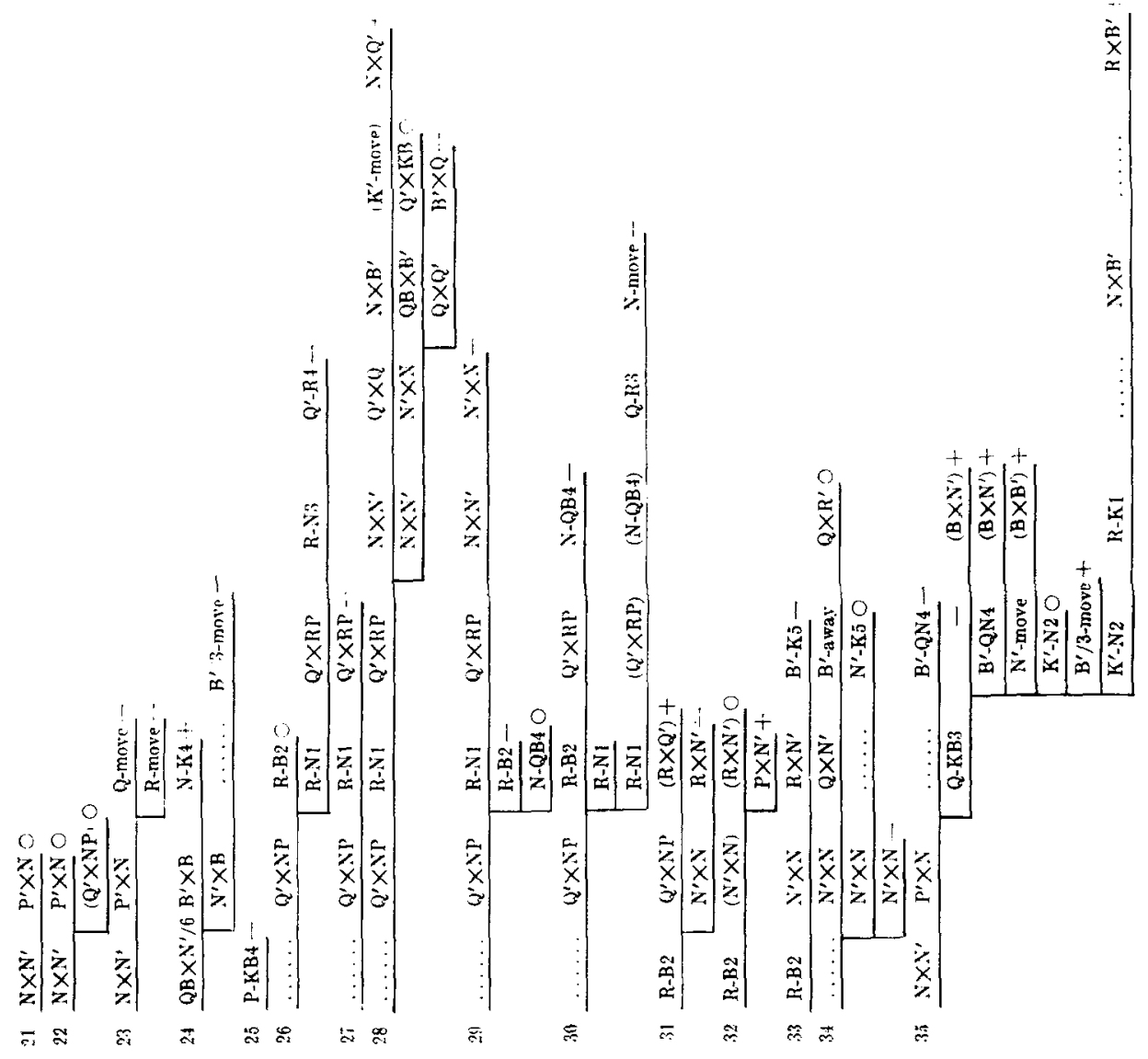




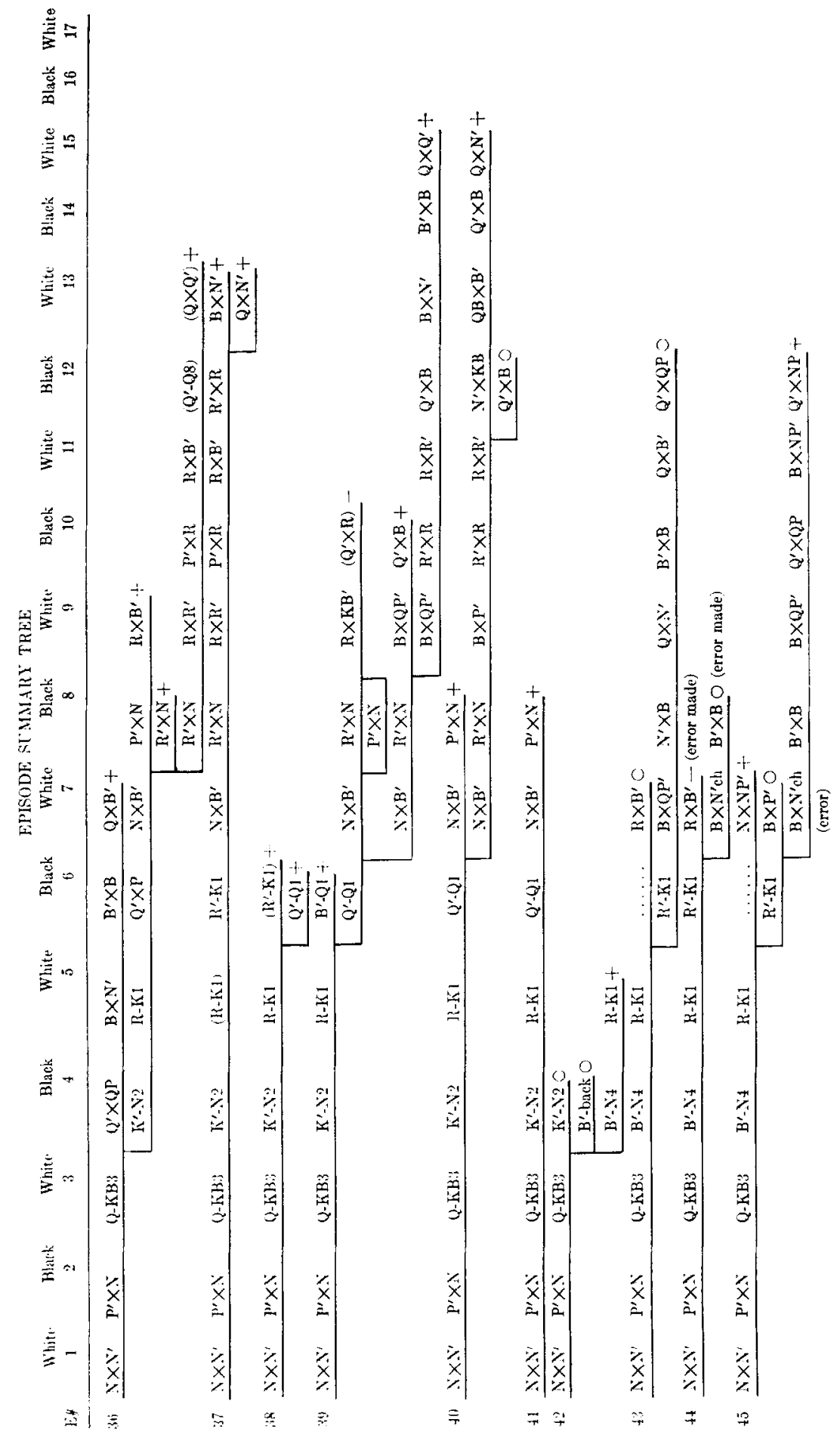

